\title{
Time Evolution of the Magnetic Chains with Uniaxial Anisotropy
}

\author{
D. WOŹNIAK ${ }^{a}$, A. DRZEWIŃSKI ${ }^{a *}$ AND G. KAMIENIARZ ${ }^{b}$ \\ ${ }^{a}$ Institute of Physics, University of Zielona Góra, Prof. Z. Szafrana 4a, 65-516 Zielona Góra, Poland \\ ${ }^{b}$ Faculty of Physics, A. Mickiewicz University, Umultowska 85, 61-614 Poznań, Poland
}

\begin{abstract}
In the framework of the matrix product states representation the effect of the single-ion anisotropy on the time evolution of the initial state after a sudden quench of the local magnetic field has been investigated. The overlap of the initial and time-evolved states, so called the Loschmidt echo, magnetization profiles and correlation functions are presented.
\end{abstract}

DOI: 10.12693 /APhysPolA.127.333

PACS: 75.10.Jm, 76.60.Es, 05.30.-d

\section{Introduction}

Quantum magnetism is one of scientific disciplines where experimental and theoretical studies strongly stimulate each other. Recent unprecedented progress in the study of ultracold gases has opened up for experimental studies of spin models with an incomparable accuracy. Atoms with the desired spin are placed in nodes of optical lattices where their interaction can be tuned by external fields [1]. It opens the experimental way to investigate the ground state and dynamical properties of magnetic chains.

In the present paper, the one-dimensional spin-1 antiferromagnetic Heisenberg model is investigated by the matrix product states (MPS) formalism [2]. To study the ground-state magnetization dynamics we have considered the chain with $N=100$ sites and open boundary conditions. The $S=1$ Heisenberg Hamiltonian consists of an isotropic exchange term and uniaxial single-ion anisotropy term

$$
\mathcal{H}=J \sum_{i=1}^{N-1} \boldsymbol{S}_{i} \boldsymbol{S}_{i+1}+D \sum_{i=1}^{N}\left(S_{i}^{z}\right)^{2},
$$

where $\boldsymbol{S}_{i} \boldsymbol{S}_{i+1}=S_{i}^{x} S_{i+1}^{x}+S_{i}^{y} S_{i+1}^{y}+S_{i}^{z} S_{i+1}^{z}, \quad J$ is an exchange coupling constant and $D$ is the uniaxial anisotropy parameter.

We consider two types of quantum quenches: the central spins perturbation and the whole chain perturbation. For $t \leq 0$ the Hamiltonian is extended by the additional term taking one of the following form:

$$
\begin{aligned}
& \mathcal{H}(t \leq 0)=\mathcal{H}+B\left(S_{N / 2}^{z}-S_{N / 2+1}^{z}\right), \\
& \mathcal{H}(t \leq 0)=\mathcal{H}+B\left(\sum_{i=1}^{N / 2} S_{i}^{z}-\sum_{i=N / 2+1}^{N} S_{i}^{z}\right) .
\end{aligned}
$$

The parameter $B$ is a local magnetic field acting along the $z$-th coordinate axis and for simplicity the $g$-factors and $\mu_{B}$ have been set to one. To be in the high-field

* corresponding author; e-mail: A.Drzewinski@if.uz.zgora.pl regime $B$ was set to $5 J$. The initial state $\left|\psi_{0}\right\rangle$ is chosen as the ground state of $\mathcal{H}(t \leq 0)$ and then for $t>0$ the time evolution is governed by the Hamiltonian $\mathcal{H}$. An initial quantum state $\left|\psi_{0}\right\rangle$ evolves into a state

$$
|\psi(t)\rangle=\exp (-\mathrm{i} \mathcal{H} t)\left|\psi_{0}\right\rangle \text {. }
$$

In order to measure the overlap of the time-evolved state and the initial state, the Loschmidt echo can be defined [3]:

$$
L(t)=\left|\left\langle\psi_{0}|\exp (-\mathrm{i} \mathcal{H} t)| \psi_{0}\right\rangle\right|^{2} .
$$

The infinite AF spin-1 Heisenberg chain with vanishing uniaxial anisotropy has a nonzero energy gap between the singlet ground state and the first excited state, namely the Haldane gap [4]. However, for a finite chain with open boundary conditions the ground state is split into four states converging to the same ground state as the length of the chain tends to infinity [5].

When the uniaxial anisotropy is taken into account the Haldane phase was found to exist between $D \approx-0.2$ and $D \approx 1$ [6]. In order to check if there is a relationship between the Haldane gap and relaxation dynamics of the perturbed state, we carry out calculations for three values of the parameter $D=0, \pm 1.5$.

\section{Time evolution}

The observation that for physical systems only minor part of Hilbert space is involved [7, 8], resulted in the rapid development of numerical methods based on a variational method within the space of MPS. It corresponds to assigning a finite entanglement content to spins in the ground state. Therefore, any state of the spin chain can be presented in the MPS representation

$$
|\psi\rangle=\sum_{\sigma_{1}, \ldots, \sigma_{N}}^{d_{1}, \ldots, d_{N}} \sum_{a_{1}, \ldots, a_{N-1}}^{D_{1}, \ldots, D_{N-1}} M_{1, a_{1}}^{\sigma_{1}} M_{a_{1}, a_{2}}^{\sigma_{2}} \ldots M_{a_{N-1}, 1}^{\sigma_{N}}|\boldsymbol{\sigma}\rangle,
$$

where $|\boldsymbol{\sigma}\rangle=\left|\sigma_{1}, \ldots, \sigma_{N}\right\rangle, d_{i}$ is dimension of the local base $\left\{\sigma_{i}\right\}$ at the $i$-th site whereas $D_{i}$ are related to the entanglement of neighbouring spins.

In an analogous manner any operator can be written as a matrix product operator (MPO):

$$
\mathcal{O}=\sum_{\sigma_{1}, \ldots, \sigma_{N}}^{d_{1}, \ldots, d_{N}} \sum_{\sigma_{1}^{\prime}, \ldots, \sigma_{N}^{\prime}}^{d_{1}, \ldots, d_{N}} W^{\sigma_{1} \sigma_{1}^{\prime}} W^{\sigma_{2} \sigma_{2}^{\prime}} \ldots W^{\sigma_{N} \sigma_{N}^{\prime}}|\boldsymbol{\sigma}\rangle\left\langle\boldsymbol{\sigma}^{\prime}\right| .
$$


Due to the above representation the state space grows only polynomially in the system size (not exponentially as usual). Thus, the time of calculations is significantly reduced for one-dimensional strongly correlated systems.

When the variational principle is applied, the ground state can be found very smoothly by the minimization procedure $\langle\psi|\mathcal{H}| \psi\rangle$ under the constraint $\langle\psi \mid \psi\rangle=1$ [8]. Moreover, it is one of the most attractive features of the MPS representation that the time evolution can also be performed very efficiently.

Therefore, discrete time as $t=N \Delta t$ can be used for the Hamiltonian (1), when a second-order Trotter decomposition is applied [8] the time-evolution operator can be presented as

$$
\mathrm{e}^{-\mathrm{i} \mathcal{H} \Delta t}=\mathrm{e}^{-\mathrm{i} \mathcal{H}_{\mathrm{o}} \Delta t / 2} \mathrm{e}^{-\mathrm{i} \mathcal{H}_{\mathrm{e}} \Delta t} \mathrm{e}^{-\mathrm{i} \mathcal{H}_{\mathrm{o}} \Delta t / 2}+O\left(\Delta t^{3}\right),
$$

where

$$
\begin{aligned}
& \mathcal{H}_{\mathrm{o}}=J \sum_{i=1}^{N / 2} \boldsymbol{S}_{2 i-1} \boldsymbol{S}_{2 i} \\
& \left.\mathcal{H}_{\mathrm{e}}=J \sum_{i=1}^{N / 2-1} \boldsymbol{S}\right]_{[} \mathbf{2} \boldsymbol{i} \boldsymbol{S}_{2 i+1}+D \sum_{i=1}^{N}\left(S_{i}^{z}\right)^{2} .
\end{aligned}
$$

Then the time-evolution algorithm takes a very simple form [8]: one starts from $\left|\psi_{0}\right\rangle$ and repeats the following steps:

1. Applying the MPO of the odd bonds to $|\psi(t)\rangle$;

2. Applying the MPO of the even bonds to $\mathrm{e}^{-\mathrm{i} \mathcal{H}_{\mathrm{o}} \Delta t / 2}|\psi(t)\rangle$;

3. Applying the MPO of the odd bonds to $\mathrm{e}^{-\mathrm{i} \mathcal{H}_{\mathrm{e}} \Delta t} \mathrm{e}^{-\mathrm{i} \mathcal{H}_{\mathrm{o}} \Delta t / 2}|\psi(t)\rangle$

4. Compressing the MPS $|\psi(t+\Delta t)\rangle=$ $\mathrm{e}^{-\mathrm{i} \mathcal{H}_{\mathrm{o}} \Delta t / 2} \mathrm{e}^{-\mathrm{i} \mathcal{H}_{\mathrm{e}} \Delta t} \mathrm{e}^{-\mathrm{i} \mathcal{H}_{\mathrm{o}} \Delta t / 2}|\psi(t)\rangle$ to the starting dimension.

\section{Ground-state dynamic properties}

In this section we present numerical studies of the quench dynamics of the AF spin-1 Heisenberg chain with uniaxial anisotropy.

When the anisotropy parameter is zero the Haldane phase exists. As one can see in Fig. 1, for both initial states the Loschmidt echo decays very rapidly: exponentially for the central perturbation and even faster for the whole chain perturbation. This difference is reflected also by a faster involution of the correlation function for the second case. Moreover, for the Loschmidt echo there is a distinct oscillation superimposed over the decay curve. It is worth adding that the magnetization profiles and the correlation function for other components were calculated as well. The results obtained, not presented for reasons of space, are in correspondence with the presented ones.

Next, we consider the time evolution out of the Haldane phase when $D= \pm 1.5$. For the easy-axis anisotropy (negative $D$ ), both the Loschmidt echoes strongly oscillate (see Fig. 2), the exponential decay can only be observed for the whole chain perturbation. In this case, although the spin arrangement along the $z$-th axis is
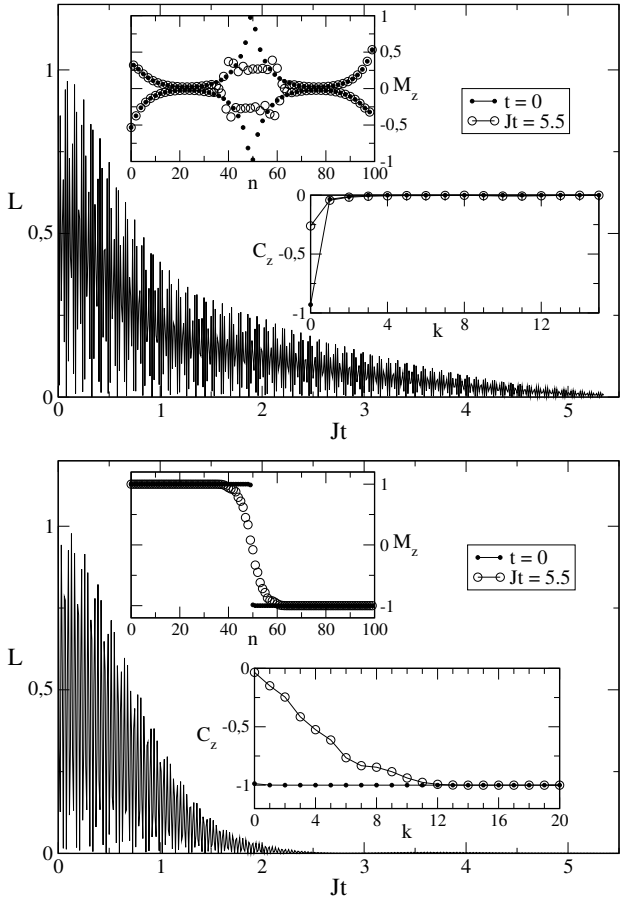

Fig. 1. The time evolution of the Loschmidt echo for the vanishing uniaxial anisotropy. The upper figure corresponds to the central spins perturbation and the bottom one to the whole chain perturbation. Upper insets present the initial and final magnetization along the $z$-th axis. Bottom insets show the initial and final correlations between the $z$-th spin components arranged symmetrically with respect to the chain center.
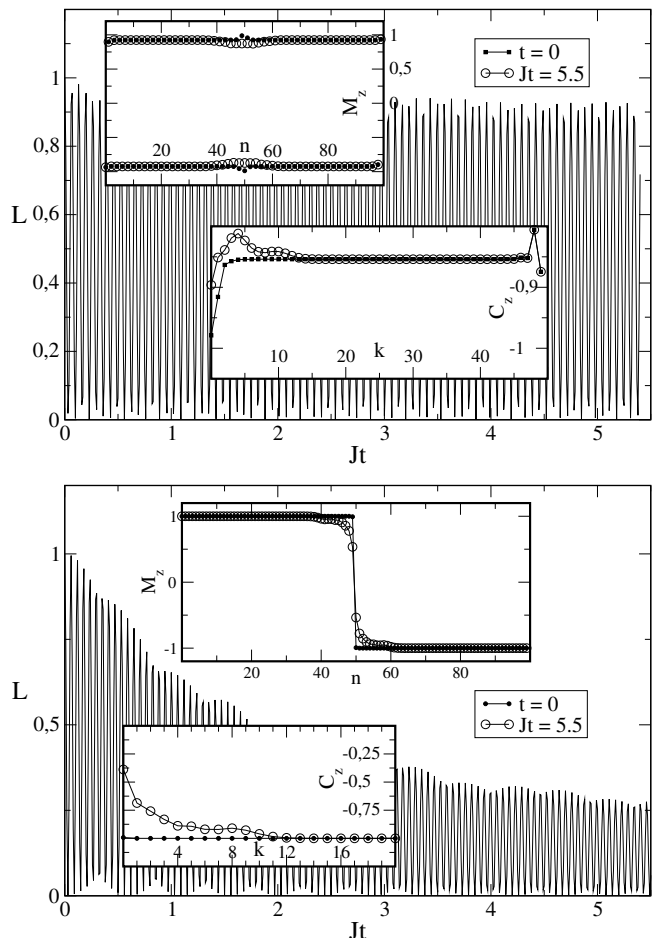

Fig. 2. The time evolution of the Loschmidt echo for the easy-axis anisotropy. Setting drawings and inserts are of the same data type as in Fig. (1). 
energetically favourable, the initial state does not take into account the antiferromagnetic interaction between nearest neighbours and it has to relax to a substantially different state. The second initial state fits both the antiferromagnetic order and the direction of the easy-axis resulting in a very weak decay of the Loschmidt echo.

For the easy-plane anisotropy (positive $D$ ) the direction of the local magnetic field, which prepares the initial state is perpendicular to the plane preferred by the uniaxial anisotropy. It makes the time evolution complex and the Loschmidt echo is seen to become less regular, involving several oscillation frequencies (see Fig. 3).
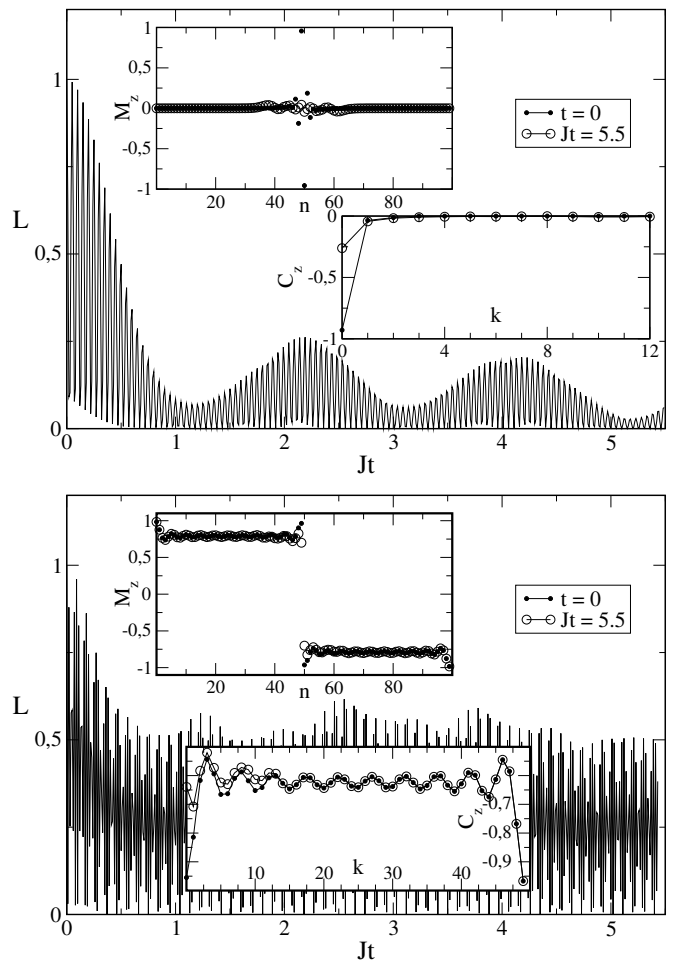

Fig. 3. The time evolution of the Loschmidt echo for the easy-plane anisotropy. Setting drawings and inserts are of the same data type as in Fig. (1).

\section{Conclusions}

We have examined the zero-temperature response of a finite AF spin-1 Heisenberg chain after the sudden change of the system parameters. The anisotropy parameter was chosen in several ways: for $D=0$ the Haldane gap was present whereas for $D= \pm 1.5$ was absent.

In order to simulate the dynamics of one-dimensional quantum systems the matrix product states representation was employed. The time evolution of the Loschmidt echo, magnetization profiles and spin-spin correlation functions have been performed.

According to our numerical results, it is difficult to say whether the differences in the time evolution of initial states are due to the presence of Haldane gap or only with a high anisotropy favouring an easy axis or easy plane. Further studies are required to provide a definitive answer.

\section{Acknowledgments}

D.W. acknowledges support as a scholar within Submeasure 8.2.2 Regional Innovation Strategies, Measure 8.2 Transfer of knowledge, Priority VIII Regional human resources for the economy Human Capital Operational Programme cofinanced by European Social Fund and state budget.

\section{References}

[1] T. Kinoshita, T. Wenger, D.S. Weiss, Nature 440 , 900 (2006); I. Bloch, J. Dalibard, W. Zwerger, Rev. Mod. Phys. 80, 885 (2008).

[2] S. Östlund, S. Rommer, Phys. Rev. Lett. 75, 3537 (1995).

[3] B. Pozsgay, J. Stat. Mech. 13, P10028 (2013).

[4] F.D. Haldane, Phys. Rev. Lett. 50, 1153 (1983).

[5] I. Affleck, T. Kennedy, E.H. Lieb, H. Tasaki, Phys. Rev. Lett. 59, 799 (1987).

[6] W. Chen, K. Hida, B.C. Sanctuary, Phys. Rev. B 67, 104401 (2003).

[7] F. Verstraete, V. Murg, K. Cirac, Adv. Phys. 57, 143 (2008).

[8] U. Schollwoeck, Ann. Phys. 326, 96 (2011). 